\title{
Multimodal collaborative activity among architectural designers using an augmented desktop at distance or in collocation
}

\author{
Jean-Marie Burkhardt ${ }^{1,3}$, Françoise Détienne ${ }^{2,3}$, Linda Moutsingua-Mpaga ${ }^{3}$, \\ Laurence Perron ${ }^{4}$, Stéphane Safin ${ }^{5}$, Pierre Leclercq \\ ${ }^{1}$ Paris Descartes University, Ergonomics-Behavior-Interaction Lab. Paris, France \\ ${ }^{2}$ LTCI CNRS-Telecom Paris Tech, Paris, France \\ ${ }^{3}$ INRIA, Rocquencourt, France \\ ${ }^{4}$ France Telecom R\&D / Orange Labs, Lannion, France \\ ${ }^{5}$ Lucid Group (Lab for User Cognition and Innovative Design) - Faculty of Applied Sciences - \\ University of Liège - Belgium
}

\begin{abstract}
Motivation - To analyse how Augmented Reality associated to video may affect collaborative design and multimodal interactions.

Research approach - An exploratory study that aims to compare 2 pairs of last year students in co-presence with 1 distant pair. Each pair had to solve an architectural design problem. Collected video has been coded with a systematic method of protocol analysis.

Findings/Design - When using an AR desktop-based $\mathrm{CAD}$, distance may not affect the design process itself whereas it may affect how the process is distributed across the various modalities of collaboration. Furthermore, collaborating and architectural experiences influence collaboration and/or design.

Research limitations/Implications - Only 3 pairs of students participated in the study resulting in $12 \mathrm{~h}$ of video protocol, which limits generalisation of the findings.

Originality/Value - The research makes a contribution in providing a detailed view on how external (e.g. situation, technology) and individual factors may affect the activity of collaborative design. Furthermore, we propose a coding method usable beyond design in a wide range of collaborative activities to underline how they are affected by technology and other situational constraints.

Take away message - Technology constraints as well as personnal characteristics of designers result in designing with specific forms of multimodal collaboration.
\end{abstract}

Copyright is held by the author/owner(s)

ECCE'08, September 16-19, 2008, Madeira, Portugal. ACM 978-1-60558-399-0/08/09

\section{Keywords}

Collaborative design, multimodal communication, empirical study, protocol analysis method

\section{OBJECTIVES}

The objective of this study is to investigate how augmented reality (AR) associated to a video media affects collaborative design activity in a distant situation compared to collocation. We specifically focus our analysis on collaborative processes and on the use of multimodal channels during interactions while performing architectural design. For that purpose, we have developed a methodological approach integrating the multimodal dimensions of communication in the analysis of interactions.

\section{USING AUGMENTED REALITY (AR) TO SUPPORT ARCHITECTURAL DESIGN: ESQUISE AND THE VIRTUAL DESKTOP}

AR was initially developed on the basis of Virtual Reality technologies. It intends to augment the user's perception and capabilities by integrating virtual objects into a real environment in real time (Azuma et al., 2001; Milgram and Kishino, 1994). Augmented desktop and tangible interfaces are frequently proposed to provide new means to support collaborative interactions and problem-solving activities (e.g. architectural design, story telling, urban planning). However, evaluations are still lacking that replicate realistic aspects of work situations and subjects close to target users population.

In order to assist architects in preliminary design while keeping the natural and simple characteristics of the pen/paper drawing process, the Lucid Group has developed an integrated aided design tool (the EsQUIsE software, Juchmes \& Leclercq, 2001; 2004; Leclercq, 2005) based on a Virtual Desktop (Figure 1). This environment is composed of a mixed software and hardware solution which offers (i) the natural aspect of 
digital freehand sketching, (ii) the ability of drawing interpretation and generating a 3D model and (iii) the direct model manipulation and evaluation of performances (presently in building engineering). The system consists in a classical "A0" desk with a suspended ceiling equipped with a double projection system offering a large working surface (approximately $150 \times 60 \mathrm{~cm})$. The electronic stylus allows drawings of virtual sketches on this surface. The designer can manipulate its drawings and is provided with automatically generated models without having to use any usual modeller in the AR environment.

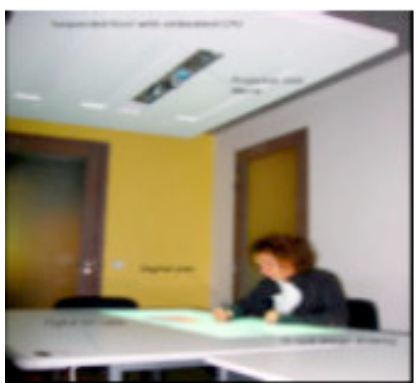

Figure 1 : EsQUIsE and The Virtual Desktop software in a single user situation

Earlier experiment (Safin et al. 2005) about Virtual Desktop's individual use have demonstrated that the immersive aspect of the system interface modifies the relationship between the designer and his/her model. The study showed how this property of the tool generates a new type of augmented interaction. The study reported in this paper is the first one to test this environment for collaborative use.

\section{DISTANT COLLABORATION AND MULTIPLE MODALITIES OF INTERACTION}

Key characteristics of collocated synchronous interactions have been identified in the literature (see for example: Olson and Olson, 2000). Rapid feedback allows for rapid corrections when there are misunderstanding or disagreements. Multiple channels (visual, oral, etc.) allow for several ways to convey complex message and provide redundancy: e.g. gaze and gestures can easily identify the referent of deictic terms. The shared local context allows for mutual understanding about what is in other's mind. More generally, those characteristics are assumed to support grounding (Clark \& Brennan, 1991) and social, situation and activity awareness (Carroll et al. 2003).

At distance, characteristics of communication media, such as no visibility, or no simultaneity (Clark and Brennan, 1991), may affect grounding and awareness.

Permission to make digital or hard copies of all or part of this work for personal or classroom use is granted without fee provided that copies are not made or distributed for profit or commercial advantage and that copies bear this notice and the full citation on the first page. To copy otherwise, or republish, to post on servers or to redistribute to lists, requires prior specific permission and/or a fee.

ECCE'08, September 16-19, 2008, Madeira, Portugal.

Copyright 2008 ACM 978-1-60558-399-0/08/09...\$5.00.
Using videoconferencing tools can extend the channels by which people communicate.

In our study, we have combined our AR setting with a videoconferencing media to allow designers to collaborate through multiple channels and modalities. In this situation, our research questions concern how particular constraints of these technologies may affect the ease of interaction management activities and, more specifically, may transform the nonverbal and verbal conducts or the modalities used to collaborate. Finally, we are also interested in examining whether these various constraints may affect the design activity itself. Compared to previous ethnography-oriented studies, the originality of our study is to have developed and used a coding scheme allowing a fine-grained analysis of how technology differentially affects the collaborative activity and a vocational activity, i.e. design.

\section{METHOD}

The participants are three pairs of last year students (from 22 to 24 years old) in architecture or in building engineering and architecture. They have a similar experience in design tasks, to avoid biases due to the diversity of professional practices, particularly important in architecture.

Two pairs (P1 and P2) were in co-presence: both designers of each pair were sit side by side on the virtual desktop. One pair (P3) was at distance: each designer of a pair had at his/her disposal a graphics tablet, a webcam to see the other member of the pair and an audio channel for verbal exchange. Each pair had specific particularities summarized in Table 1 . The collaborating experience refers to the previous experience of each pair in collaborating together.

\begin{tabular}{|l|l|l|l|}
\hline Pairs & $\begin{array}{l}\text { Experimental } \\
\text { setting }\end{array}$ & Curriculum & $\begin{array}{l}\text { Collaborating } \\
\text { experience }\end{array}$ \\
\hline P1 & Co-presence & Architecture & No familiar \\
\hline P2 & Co-presence & Engineering-architecture & Familiar \\
\hline P3 & Distant & Engineering-architecture & No familiar \\
\hline
\end{tabular}

\section{Table 1: Pairs specificities}

Each pair's task was to solve together a design problem. The exercise consists in the preliminary design of a school. The building constraints (technical, functional, urban...) are usual and are synthesized in a program document. This task is representative of real architectural design tasks but, as it does not require professional complex management resources, it is appropriate to students. Furthermore, as students have been regularly confronted to this kind of design tasks during 5 years, they can be seen as experts for this task. The experimental settings were as follows.

Co-presence condition (Figure 2): the two designers of P1 and P2 work together on a single Virtual Desktop with EsQUIsE software. Due to technical constraints, although they each have a stylus, they are not allowed to use them at the same time.

Distant condition (Figure 3): the designers of P3 are working on two screen-tablets (Wacom Cintiq) on 
which runs the EsQUIsE Software. The two tablets are remotely connected, so the drawing is shared among the two tablets. To complete this setting, a "classical" videoconference software (iChat) is running on a separate screen.

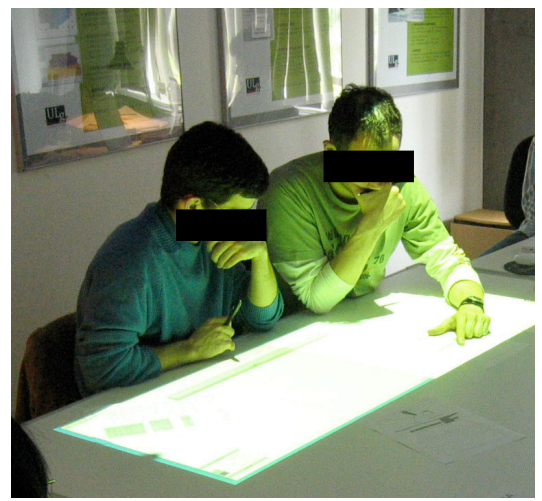

Figure 2 Co-presence condition : a pair of designers on the same Virtual Desktop

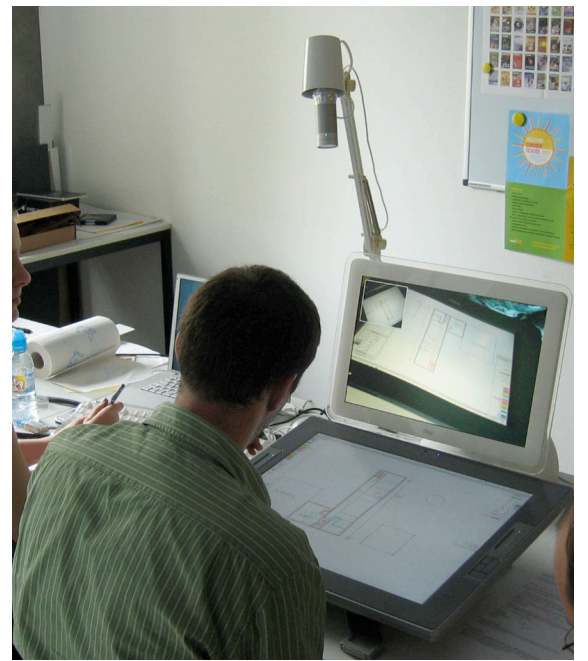

Figure 3 Distant condition : one designer of a pair in front of a screen-tablet and a videoconference screen.

We have collected 12 hours of video corresponding to the three pairs interactions. They have been transcribed and coded with the categories from an analysis methodology (inspired from Détienne et al. 2004; Détienne et al. 2006; Green, 2004) that distinguishes: design actions (exploratory comprehension, incrementation, modification, transcription, search of information); collaborative actions (co-realisation of actions, planning, resources management, metacognitive regulation, discourse regulation); modalities used (verbal, textual, graphical, visual/gestural, physical). The resulting data have been analysed with statistical descriptive methods (Cramer's V, Relative Deviations).

\section{MAIN RESULTS}

First, the main categories of activities related to design show a similar distribution across the pairs. This result suggests that the design process is similar whatever the localisation of participants. Futhermore, the main modality of interaction was verbal whatever the collocated vs. distant situation which is consistent with previous studies (see for example Heath \& Luff, 1991).

The second main results concern new functions of modalities of interaction observed at distance linked to difficulties for sharing context. In co-presence, both designers share the same physical space and can exploit the corresponding range of modalities to communicate and to acquire information in the course action. We found that distance matters, in particular to construct and maintain a shared context and to construct awareness of the other actions and intentions.

Indeed, in the distance situation, there are two main constraints to communicate and interact: partial visibility and no simultaneity. With the webcam and the video displayed on a small monitor, each member of the distant pair has a partial visibility of the working space of the other. By default, they have the other face displayed, but they can adjust the webcam to see the other working space. Even so, their visibility remains partial. Furthermore, the transmission of any entries in the RA system is not simultaneous. In particular, when a drawing or textual entry is done, the transmission is effective when the entry is finished (the stylet is up).

Difficulties to construct shared context are revealed by verbal or textual exchanges of $\mathrm{P} 3$ to ensure that both members share the same visual context. As the designers have no visual control on the working visual context of the other (partial visibility), and that the system is not completely synchronous (no simultaneity), they check regularly mainly by verbal exchanges what the other is seeing or looking at. Furthermore, we observed verbal or textual exchanges of P3 to check the other graphical actions or intentions. For the same reasons as previously, partial visibility and no simultaneity, it is difficult to follow the other actions or intentions. However, this is particularly important as the data entries have to be sequential (even if each member of the pairs has his/her own stylet, graphical or textual entries have to be done sequentially). Thus, each designer checks regularly the other's actions or intentions, mainly by verbal exchanges. Distance also matters to let the other see gestural simulations or gestural referencing. We observed that, for P3 at distance, graphical actions replace these gestural actions, as performed by $\mathrm{P} 1$ and P2 in co-presence. Indeed, partial visibility prevents gestural actions to be seen at distance. Distance also prevents co-realisation of graphical actions (e.g. one draw a line while the other holds a rule; one describes a drawing while the other performs the sketch) as it occurs in co-presence for P1 and P2. These results are summarized in table 2 . 


\begin{tabular}{|l|l|l|}
\hline Activity/goal & Co-located pair & Distant pair \\
\hline $\begin{array}{l}\text { Synchr. current } \\
\text { area of interest }\end{array}$ & $\begin{array}{l}\text { Visual and physical } \\
\text { sharing of space }\end{array}$ & $\begin{array}{l}\text { Verbal / textual } \\
\text { iterative controls }\end{array}$ \\
\cline { 1 - 2 } Synchr. actions & \multicolumn{2}{|c|}{ Verbal interactions } \\
\hline $\begin{array}{l}\text { Design } \\
\text { exploration }\end{array}$ & $\begin{array}{l}\text { Gesture associated to } \\
\text { verbal }\end{array}$ & $\begin{array}{l}\text { Graphical expression } \\
\text { via pointer } \\
\text { associated to verbal }\end{array}$ \\
\hline $\begin{array}{l}\text { simulation of } \\
\text { design solution }\end{array}$ &
\end{tabular}

Table 2: co-location / distance effect on pair activity

We found also specific uses of the virtual desktop related to previous collaborating and architectural experience. The pair P2, who had previous experience in collaborating together, had a more explicit and efficient planning activity and was the only one in copresence to explicitly divide the virtual desktop into public and private spaces to optimise the design process. This seems to participate in optimising the design process, as revealed by less transcriptions and less modifications.

The pair P1 had a curriculum in architecture different from P2 and P3 who were formed both in engineering and architecture. We observed that P1 was the pair who gave greater importance to graphical actions, and to the shift from 2D to 3D.

\section{CONCLUSION AND PERSPECTIVES}

Our methodology, based on a scheme of coding, has enabled us to make clear how technology constraints affect collaborative activity at distance.

This methodology is generic enough to be applied to other collaborative problem solving activities and other technology-mediated situations. While our study does not show evidence of effects of distance on the design process with augmented reality, it reveals that technology-mediation transforms the modalities used to collaborate. Indeed, looking deeper at the multimodal dimensions of activity has underlined function exchanges between modalities like verbal to graphics for coordination. While these observations reveal forms of adaptation of collaboration processes with respect to technology constraints, a pending question to be discussed is the collaborative effort it implies.

Furthermore, our results tend to show that collaborating and architectural experiences influence collaboration and/or design which provides evidence to formulate more detailed hypotheses about the effect of acquaintance on designers' activity.

Practically, our results will support the specification of desirable properties of the Virtual desktop to support collaboration which have been implemented in the BV and a second experiment will be carried out with an improved AR collaborative system that takes into account usability aspects related to supporting collaboration identified in this first experiment.

\section{REFERENCES}

Azuma RT, Baillot Y, Behringer R, Feiner S, Julier S, MacIntyre B. (2001). Recent advances in augmented reality. IEEE Computer Graphics and Applications, 21, 34-47.

Carroll, J. M., Neale, D. C., Isenhour, P. L., Rosson, M. B. \& McCrickard, D. S. (2003) Notification and awareness: synchronizing task-oriented collaborative activity. International Journal of Human-Computer Studies, 58(5), 605-632.

Clark, H.H. \& Brennan, S. E. (1991) Grounding in communication. In L. Resnick, J-M Levine \& S. D. Teasley (Eds) : Perspectives on socially shared cognition. (APA, Washington DC) 127149.

Détienne, F., Boujut, J-F., \& Hohmann, B. (2004) Characterization of Collaborative Design and Interaction Management Activities in a Distant Engineering Design Situation. In F. Darses, R.. Dieng, C. Simone, M. Zaklad (Eds) Cooperative Systems design. IOS Press, 83- 98.

Détienne, F., Visser, W., \& Tabary, R. (2006) Articulation des dimensions graphico-gestuelle et verbale dans l'analyse de la conception collaborative. Psychologie de l'Interaction, 2122, 283-308.

Green, T. R. G. (2000) Instructions and descriptions: some cognitive aspects of programming and similar activities. Invited paper, in Di Gesù, V., Levialdi, S. and Tarantino, L., (Eds.) Proceedings of Working Conference on Advanced Visual Interfaces (AVI 2000). New York: ACM Press, pp 21-28.

Heath, C. \& Luff, P. (1991) Disembodied conduct: communication through video in a multi-media office environment. Proceedings of the SIGCHI conference on Human factors in computing systems: Reaching through technology. CHI 1991, New Orleans, Louisiana, US. 99 - 103.

Juchmes R., \& Leclercq P. (2004) A Multi-Agent System for Architectural Sketches Interpretation, Eurographics Workshop on Sketch-Based Interfaces, Grenoble, France.

Leclercq, P. (2005) Le concept d'esquisse augmentée. $S C A N^{\prime} 05$, Paris, Ecole Nationale Supérieure d'Architecture de Paris-Val de Seine, France.

Milgram P, Kishino F. (1994) A taxonomy of mixed reality visual displays. IEICE Transactions on Information Systems, 12:1321-9.

Olson, G.M., \& Olson, J.S. (2000) Distance matters. Human-Computer Interaction, 15, 139-178.

Safin S., Boulanger C. \& Leclercq P. (2005) Premières évaluations d'un Bureau Virtuel pour un processus de conception augmenté. 17ème Conférence Francophone sur l'Interaction Homme-Machine, Toulouse, France 